DOI: https://doi.org/10.15688/mpcm.jvolsu.2017.3.7

UDC 517

LBC 22.161

\title{
EQUIVALENCE OF RECURRENCE AND LIOUVILLE PROPERTY FOR SYMMETRIC DIRICHLET FORMS ${ }^{1}$
}

\author{
Naotaka Kajino \\ Associate Professor, Department of Mathematics, Graduate School of Science, \\ Kobe University \\ nkajino@math.kobe-u.ac.jp \\ Rokkodai-cho 1-1, Nada-ku, Kobe 657-8501, Japan
}

\begin{abstract}
Given a symmetric Dirichlet form $(\mathcal{E}, \mathcal{F})$ on a (non-trivial) $\sigma$-finite measure space $(E, \mathcal{B}, m)$ with associated Markovian semigroup $\left\{T_{t}\right\}_{t \in(0, \infty)}$, we prove that $(\mathcal{E}, \mathcal{F})$ is both irreducible and recurrent if and only if there is no non-constant $\mathcal{B}$-measurable function $u: E \rightarrow[0, \infty]$ that is $\mathcal{E}$-excessive, i.e., such that $T_{t} u \leq u m$-a.e. for any $t \in(0, \infty)$. We also prove that these conditions are equivalent to the equality $\left\{u \in \mathcal{F}_{e} \mid \mathcal{E}(u, u)=0\right\}=\mathbb{R} \mathbf{1}$, where $\mathcal{F}_{e}$ denotes the extended Dirichlet space associated with $(\mathcal{E}, \mathcal{F})$. The proof is based on simple analytic arguments and requires no additional assumption on the state space or on the form. In the course of the proof we also present a characterization of the $\mathcal{E}$-excessiveness in terms of $\mathcal{F}_{e}$ and $\mathcal{E}$, which is valid for any symmetric positivity preserving form.
\end{abstract}

Key words: symmetric Dirichlet forms, symmetric positivity preserving forms, extended Dirichlet space, excessive functions, recurrence, Liouville property.

\section{Introduction and the statement of the main theorem}

Since the classical theorem of Liouville saying that there is no non-constant bounded holomorphic function on $\mathbb{C}$, non-existence of non-constant bounded (super-)harmonic functions on the whole space, so-called Liouville property, has been one of the main concerns of harmonic analysis on various spaces. One of the most well-known facts about Liouville $\widehat{A}$ property is that the non-existence of non-constant bounded superharmonic functions on the whole space is equivalent to the recurrence of the corresponding stochastic process. Such an equivalence is known to hold for standard processes on locally compact separable metrizable spaces by Blumenthal and Getoor [1, Chapter II, (4.22)] and also for more general right processes by Getoor [9, Proposition (2.4)]. Getoor [8, Proposition 2.14] provides the same kind of equivalence in terms of excessive measures. The purpose of this paper is to 
give a completely elementary proof of this equivalence in the framework of an arbitrary symmetric Dirichlet form on a (non-trivial) $\sigma$-finite measure space. Our proof is purely functional-analytic and free of topological notions on the state space, although we need to assume the symmetry of the Dirichlet form.

In the rest of this section, we describe our setting and state the main theorem. We fix a $\sigma$-finite measure space $(E, \mathcal{B}, m)$ throughout this paper, and below all $\mathcal{B}$-measurable functions are assumed to be $[-\infty, \infty]$-valued. Let $(\mathcal{E}, \mathcal{F})$ be a symmetric Dirichlet form on $L^{2}(E, m)$ and let $\left\{T_{t}\right\}_{t \in(0, \infty)}$ be its associated Markovian semigroup on $L^{2}(E, m)$. Let $L_{+}(E, m):=\{f \mid f: E \rightarrow[0, \infty], f$ is $\mathcal{B}$-measurable $\}$ and $L^{0}(E, m):=\{f \mid f: E \rightarrow$ $\rightarrow \mathbb{R}, f$ is $\mathcal{B}$-measurable $\}$, where we of course identify any two $\mathcal{B}$-measurable functions which are equal $m$-a.e. Let $\mathbf{1}$ denote the constant function $\mathbf{1}: E \rightarrow\{1\}$, and we regard $\mathbb{R} \mathbf{1}:=\{c \mathbf{1} \mid c \in \mathbb{R}\}$ as a linear subspace of $L^{0}(E, m)$. Also let $L_{+}^{p}(E, m):=L^{p}(E, m) \cap$ $\cap L_{+}(E, m)$ for $p \in[1, \infty] \cup\{0\}$. Note that $T_{t}$ is canonically extended to an operator on $L_{+}(E, m)$ and also to a linear operator from $\mathcal{D}\left[T_{t}\right]:=\left\{f \in L^{0}(E, m)\left|T_{t}\right| f \mid<\infty m\right.$-a.e. $\}$ to $L^{0}(E, m)$; see Proposition 1 below.

Definition 1. $u \in L_{+}(E, m)$ is called $\mathcal{E}$-excessive if and only if $T_{t} u \leq u m$-a.e. for any $t \in(0, \infty)$. Similarly, $u \in \bigcap_{t \in(0, \infty)} \mathcal{D}\left[T_{t}\right]$ is called $\mathcal{E}$-excessive in the wide sense if and only if $T_{t} u \leq u m$-a.e. for any $t \in(0, \infty)$.

Remark 1. As stated in $[1 ; 2 ; 6 ; 7 ; 14]$, when we call a function $u$ excessive, it is usual to assume that $u$ is non-negative, which is why we have added "in the wide sense" in the latter part of Definition 1 .

$\mathcal{E}$-excessive functions will play the role of superharmonic functions on the whole state space, and the main theorem of this paper (Theorem 1) asserts that $(\mathcal{E}, \mathcal{F}$ ) is irreducible and recurrent if and only if there is no non-constant $\mathcal{E}$-excessive function.

Yet another possible way of formulation of harmonicity of functions (on the whole space $E$ ) is to use the extended Dirichlet space $\mathcal{F}_{e}$ associated with $(\mathcal{E}, \mathcal{F}) ; u \in \mathcal{F}_{e}$ could be called "superharmonic" if $\mathcal{E}(u, v) \geq 0$ for any $v \in \mathcal{F}_{e} \cap L_{+}(E, m)$, and "harmonic" if $\mathcal{E}(u, v)=0$ for any $v \in \mathcal{F}_{e}$, or equivalently, if $\mathcal{E}(u, u)=0$. In fact, as a key lemma for the proof of the main theorem, in Proposition 3 below we prove that $u \in \mathcal{F}_{e}$ is "superharmonic" in this sense if and only if $u$ is $\mathcal{E}$-excessive in the wide sense. Under this formulation of harmonicity, if $(\mathcal{E}, \mathcal{F})$ is recurrent, i.e., $\mathbf{1} \in \mathcal{F}_{e}$ and $\mathcal{E}(\mathbf{1}, \mathbf{1})=0$, then the non-existence of non-constant harmonic functions amounts to the equality

$$
\left\{u \in \mathcal{F}_{e} \mid \mathcal{E}(u, u)=0\right\}=\mathbb{R} \mathbf{1}
$$

Ōshima [10, Theorem 3.1] proved (1.1) (and the completeness of $\left(\mathcal{F}_{e} / \mathbb{R} \mathbf{1}, \mathcal{E}\right)$ as well) for the Dirichlet form associated with a symmetric Hunt process which is recurrent in the sense of Harris; note that the recurrence in the sense of Harris is stronger than the usual recurrence of the associated Dirichlet form. Fukushima and Takeda [7, Theorem 4.2.4] (see also [2, Theorem 2.1.11]) showed (1.1) for irreducible recurrent symmetric Dirichlet forms $(\mathcal{E}, \mathcal{F})$ under the (only) additional assumption that $m(E)<\infty$. In the recent book [2], Chen and Fukushima has extended this result to the case of $m(E)=\infty$ when $(\mathcal{E}, \mathcal{F})$ is regular, by using the theory of random time changes of Dirichlet spaces. As part of our main theorem, we generalize (1.1) to any irreducible recurrent symmetric Dirichlet form. In fact, this generalization could be obtained (at least when $L^{2}(E, m)$ is separable) also by applying the theory of regular representations of Dirichlet spaces (see [6, Section A.4]) to 
reduce the proof to the case where $(\mathcal{E}, \mathcal{F})$ is regular. The advantage of our proof is that it is based on totally elementary analytic arguments and is free from any use of time changes or regular representations of Dirichlet spaces.

Here is the statement of our main theorem. See [2, Section 1.1] or [4, Section 1] for basics on $\mathcal{F}_{e}$, and [6, Sections 1.5 and 1.6] or [2, Section 2.1] for details about irreducibility and recurrence of $(\mathcal{E}, \mathcal{F})$. We remark that $\mathcal{F}_{e} \subset \bigcap_{t \in(0, \infty)} \mathcal{D}\left[T_{t}\right]$ by Lemma 2-(1) below. We say that $(E, \mathcal{B}, m)$ is non-trivial if and only if both $m(A)>0$ and $m(E \backslash A)>0$ hold for some $A \in \mathcal{B}$, which is equivalent to the condition that $L^{2}(E, m) \not \subset \mathbb{R} \mathbf{1}$ since $(E, \mathcal{B}, m)$ is assumed to be $\sigma$-finite.

Theorem 1. Consider the following six conditions.

1) $(\mathcal{E}, \mathcal{F})$ is both irreducible and recurrent.

2) $\left\{u \in \mathcal{F}_{e} \mid \mathcal{E}(u, u)=0\right\}=\mathbb{R} \mathbf{1}$.

3) $\left\{u \in \mathcal{F}_{e} \cap L_{+}^{\infty}(E, m) \mid \mathcal{E}(u, u)=0\right\}=\{c \mathbf{1} \mid c \in[0, \infty)\}$.

4) If $u \in \mathcal{F}_{e}$ is $\mathcal{E}$-excessive in the wide sense then $u \in \mathbb{R} \mathbf{1}$.

5) If $u \in L_{+}^{0}(E, m)$ is $\mathcal{E}$-excessive then $u \in \mathbb{R} \mathbf{1}$.

6) If $u \in \mathcal{F}_{e} \cap L_{+}^{\infty}(E, m)$ is $\mathcal{E}$-excessive then $u \in \mathbb{R} \mathbf{1}$.

The three conditions $\mathbf{1}), \mathbf{2}), \mathbf{3})$ are equivalent to each other and imply 4), 5), 6). If $(E, \mathcal{B}, m)$ is non-trivial, then the six conditions are all equivalent.

The organization of this paper is as follows. In Section 2, we prepare basic results about the extended space $\mathcal{F}_{e}$ and $\mathcal{E}$-excessive functions, which are valid as long as $(\mathcal{E}, \mathcal{F})$ is a symmetric positivity preserving form. The key results there are Propositions 3 and 4 , which are essentially known but seem new in the present general framework. Furthermore Proposition 4 provides a characterization of the notion of $\mathcal{E}$-excessive functions in terms of $\mathcal{F}_{e}$ and $\mathcal{E}$. Making use of these two propositions, we show Theorem 1 in Section 3.

\section{Preliminaries: the extended (Dirichlet) space and excessive functions}

As noted in the previous section, we fix a $\sigma$-finite measure space $(E, \mathcal{B}, m)$ throughout this paper, and all $\mathcal{B}$-measurable functions are assumed to be $[-\infty, \infty]$-valued. Note that by the $\sigma$-finiteness of $(E, \mathcal{B}, m)$ we can take $\eta \in L^{1}(E, m) \cap L^{\infty}(E, m)$ such that $\eta>0 m$-a.e.

Notation. (0) We follow the convention that $\mathbb{N}=\{1,2,3, \ldots\}$, i.e., $0 \notin \mathbb{N}$.

(1) For $a, b \in[-\infty, \infty]$, we write $a \vee b:=\max \{a, b\}, a \wedge b:=\min \{a, b\}, a^{+}:=a \vee 0$ and $a^{-}:=-(a \wedge 0)$. For $\left\{a_{n}\right\}_{n \in \mathbb{N}} \subset[-\infty, \infty]$ and $a \in[-\infty, \infty]$, we write $a_{n} \uparrow a$ (resp. $\left.a_{n} \downarrow a\right)$ if and only if $\left\{a_{n}\right\}_{n \in \mathbb{N}}$ is non-decreasing (resp. non-increasing) and $\lim _{n \rightarrow \infty} a_{n}=a$. We use the same notation also for ( $m$-equivalence classes of) $[-\infty, \infty]$-valued functions.

(2) As introduced before Definition 1, identifying any two $\mathcal{B}$-measurable functions that are equal $m$-a.e., we set $L_{+}(E, m):=\{f \mid f: E \rightarrow[0, \infty], f$ is $\mathcal{B}$-measurable $\}, L^{0}(E, m):=$ $:=\{f \mid f: E \rightarrow \mathbb{R}, f$ is $\mathcal{B}$-measurable $\}$ and $L_{+}^{p}(E, m):=L^{p}(E, m) \cap L_{+}(E, m), p \in$ $\in[1, \infty] \cup\{0\}$. We regard $\mathbb{R} \mathbf{1}:=\{c \mathbf{1} \mid c \in \mathbb{R}\}$ as a linear subspace of $L^{0}(E, m)$. Let $\|\cdot\|_{p}$ denote the norm of $L^{p}(E, m)$ for $p \in[1, \infty]$. Finally, let $\langle f, g\rangle:=\int_{E} f g d m$ for $f, g \in L_{+}(E, m)$ and also for $f, g \in L^{0}(E, m)$ with $f g \in L^{1}(E, m)$.

Recall the following definitions regarding bounded linear operators on $L^{2}(E, m)$.

Definition 2. Let $T: L^{2}(E, m) \rightarrow L^{2}(E, m)$ be a bounded linear operator on $L^{2}(E, m)$.

(1) $T$ is called positivity preserving if and only if $T f \geq 0 \mathrm{~m}$-a.e. for any $f \in L_{+}^{2}(E, m)$.

(2) $T$ is called Markovian if and only if $0 \leq T f \leq 1 m$-a.e. for any $f \in L^{2}(E, m)$ with $0 \leq f \leq 1 m$-a.e. 
Clearly, if $T$ is positivity preserving then so is its adjoint $T^{*}$. Note that if $T$ is Markovian, then it is positivity preserving, $\|T f\|_{\infty} \leq\|f\|_{\infty}$ for any $L^{2}(E, m) \cap L^{\infty}(E, m)$ and $\left\|T^{*} f\right\|_{1} \leq\|f\|_{1}$ for any $f \in L^{1}(E, m) \cap L^{2}(E, m)$. Moreover, using the $\sigma$-finiteness of $(E, \mathcal{B}, m)$, we easily have the following proposition.

Proposition 1. Let $T: L^{2}(E, m) \rightarrow L^{2}(E, m)$ be a positivity preserving bounded linear operator on $L^{2}(E, m)$.

(1) $\left.T\right|_{L_{+}^{2}(E, m)}$ uniquely extends to a map $T: L_{+}(E, m) \rightarrow L_{+}(E, m)$ such that $T f_{n} \uparrow T f$ $m$-a.e. for any $f \in L_{+}(E, m)$ and any $\left\{f_{n}\right\}_{n \in \mathbb{N}} \subset L_{+}(E, m)$ with $f_{n} \uparrow f m$-a.e. Moreover, let $f, g \in L_{+}(E, m)$ and $a \in[0, \infty]$. Then $T(f+g)=T f+T g, T(a f)=a T f,\langle T f, g\rangle=$ $=\left\langle f, T^{*} g\right\rangle$, and if $f \leq g m$-a.e. then $T f \leq T g m$-a.e.

(2) Let $\mathcal{D}[T]:=\left\{f \in L^{0}(E, m)|T| f \mid<\infty\right.$ m-a.e. $\}$. Then $T: L^{2}(E, m) \rightarrow L^{2}(E, m)$ is extended to a linear operator $T: \mathcal{D}[T] \rightarrow L^{0}(E, m)$ given by $T f:=T\left(f^{+}\right)-T\left(f^{-}\right)$, $f \in \mathcal{D}[T]$, so that it has the following properties:

(i) If $f, g \in \mathcal{D}[T]$ and $f \leq g m$-a.e. then $T f \leq T g m$-a.e.

(ii) If $\left\{f_{n}\right\}_{n \in \mathbb{N}} \subset \mathcal{D}[T]$ and $f, g \in \mathcal{D}[T]$ satisfy $\lim _{n \rightarrow \infty} f_{n}=f$ m-a.e. and $\left|f_{n}\right| \leq|g|$ m-a.e. for any $n \in \mathbb{N}$, then $\lim _{n \rightarrow \infty} T f_{n}=T f m$-a.e.

Throughout the rest of this paper, we fix a closed symmetric form $(\mathcal{E}, \mathcal{F})$ on $L^{2}(E, m)$ together with its associated symmetric strongly continuous contraction semigroup $\left\{T_{t}\right\}_{t \in(0, \infty)}$ and resolvent $\left\{G_{\alpha}\right\}_{\alpha \in(0, \infty)}$ on $L^{2}(E, m)$; see [6, Chapter 1.3] for basics on closed symmetric forms on Hilbert spaces and their associated semigroups and resolvents.

Let us further recall the following definition.

Definition 3. (1) $(\mathcal{E}, \mathcal{F})$ is called a positivity preserving form if and only if $u^{+} \in \mathcal{F}$ and $\mathcal{E}\left(u^{+}, u^{+}\right) \leq \mathcal{E}(u, u)$ for any $u \in \mathcal{F}$, or equivalently, $T_{t}$ is positivity preserving for any $t \in(0, \infty)$.

(2) $(\mathcal{E}, \mathcal{F})$ is called a Dirichlet form if and only if $u^{+} \wedge 1 \in \mathcal{F}$ and $\mathcal{E}\left(u^{+} \wedge 1, u^{+} \wedge 1\right) \leq \mathcal{E}(u, u)$ for any $u \in \mathcal{F}$, or equivalently, $T_{t}$ is Markovian for any $t \in(0, \infty)$.

See, e.g., [11, Section 2] for the equivalences stated in Definition 3.

In the rest of this section, we assume that $(\mathcal{E}, \mathcal{F})$ is a positivity preserving form. The following definition is standard (see [12, Definition 3], [2, Definition 1.1.4] or [4, Definition $1.4])$.

Definition 4. We define the extended space $\mathcal{F}_{e}$ associated with $(\mathcal{E}, \mathcal{F})$ by

$$
\mathcal{F}_{e}:=\left\{\begin{array}{l|l}
u \in L^{0}(E, m) & \begin{array}{l}
\lim _{n \rightarrow \infty} u_{n}=u m \text {-a.e. for some }\left\{u_{n}\right\}_{n \in \mathbb{N}} \subset \mathcal{F} \\
\text { with } \lim _{k \wedge \ell \rightarrow \infty} \mathcal{E}\left(u_{k}-u_{\ell}, u_{k}-u_{\ell}\right)=0
\end{array}
\end{array}\right\}
$$

For $u \in \mathcal{F}_{e}$, such $\left\{u_{n}\right\}_{n \in \mathbb{N}} \subset \mathcal{F}$ as in (2.1) is called an approximating sequence for $u$. When $(\mathcal{E}, \mathcal{F})$ is a Dirichlet form, $\mathcal{F}_{e}$ is called the extended Dirichlet space associated with $(\mathcal{E}, \mathcal{F})$.

Obviously $\mathcal{F} \subset \mathcal{F}_{e}$ and $\mathcal{F}_{e}$ is a linear subspace of $L^{0}(E, m)$. By virtue of [13, Proposition 2], $\mathcal{F}=\mathcal{F}_{e} \cap L^{2}(E, m)$, and for $u, v \in \mathcal{F}_{e}$ with approximating sequences $\left\{u_{n}\right\}_{n \in \mathbb{N}}$ and $\left\{v_{n}\right\}_{n \in \mathbb{N}}$, respectively, the $\operatorname{limit}_{\lim _{n \rightarrow \infty}} \mathcal{E}\left(u_{n}, v_{n}\right) \in \mathbb{R}$ exists and is independent of particular choices of $\left\{u_{n}\right\}_{n \in \mathbb{N}}$ and $\left\{v_{n}\right\}_{n \in \mathbb{N}}$, as discussed in [12, before Definition 3]. By setting $\mathcal{E}(u, v):=\lim _{n \rightarrow \infty} \mathcal{E}\left(u_{n}, v_{n}\right), \mathcal{E}$ is extended to a non-negative definite symmetric bilinear form on $\mathcal{F}_{e}$. Then it is easy to see that $\lim _{n \rightarrow \infty} \mathcal{E}\left(u-u_{n}, u-u_{n}\right)=0$ for $u \in \mathcal{F}_{e}$ and any approximating sequence $\left\{u_{n}\right\}_{n \in \mathbb{N}} \subset \mathcal{F}$ for $u$. Moreover, we have the following proposition due to Schmuland [12], which is easily proved by utilizing a version [2, Theorem A.4.1-(ii)] of the Banach - Saks theorem. 
Proposition 2 ([12, Lemma 2]). Let $u \in L^{0}(E, m)$ and $\left\{u_{n}\right\}_{n \in \mathbb{N}} \subset \mathcal{F}$ satisfy $\lim _{n \rightarrow \infty} u_{n}=$ $=u m$-a.e. and $\liminf _{n \rightarrow \infty} \mathcal{E}\left(u_{n}, u_{n}\right)<\infty$. Then $u \in \mathcal{F}_{e}, \mathcal{E}(u, u) \leq \liminf _{n \rightarrow \infty} \mathcal{E}\left(u_{n}, u_{n}\right)$, and $\liminf \operatorname{in}_{n \rightarrow \infty} \mathcal{E}\left(u_{n}, v\right) \leq \mathcal{E}(u, v) \leq \limsup _{n \rightarrow \infty} \mathcal{E}\left(u_{n}, v\right)$ for any $v \in \mathcal{F}_{e}$.

In particular, we easily see from Proposition 2 that $u^{+} \in \mathcal{F}_{e}$ and $\mathcal{E}\left(u^{+}, u^{+}\right) \leq \mathcal{E}(u, u)$ for any $u \in \mathcal{F}_{e}$.

Remark 2. For symmetric Dirichlet forms, the properties of $\mathcal{F}_{e}$ stated above are well-known and most of them are proved in the textbooks [2, Section 1.1] and [7, Section 4.1] and also in [4, Section 1]. In fact, we can verify similar results in a quite general setting; see Schmuland [12] for details.

The next proposition (Proposition 3 below) requires the following lemmas.

Lemma 1. Let $\eta \in L^{1}(E, m) \cap L^{2}(E, m)$ be such that $\eta>0$ m-a.e., and set $\|u\|_{\mathcal{F}_{e}}:=$ $:=\mathcal{E}(u, u)^{1 / 2}+\int_{E}(|u| \wedge 1) \eta d m$ for $u \in \mathcal{F}_{e}$. Then we have the following assertions:

(1) $\|u+v\|_{\mathcal{F}_{e}} \leq\|u\|_{\mathcal{F}_{e}}+\|v\|_{\mathcal{F}_{e}}$ and $\|a u\|_{\mathcal{F}_{e}} \leq(|a| \vee 1)\|u\|_{\mathcal{F}_{e}}$ for any $u, v \in \mathcal{F}_{e}$ and any $a \in \mathbb{R}$.

(2) $\mathcal{F}_{e}$ is a complete metric space under the metric $d_{\mathcal{F}_{e}}$ given by $d_{\mathcal{F}_{e}}(u, v):=\|u-v\|_{\mathcal{F}_{e}}$

Proof. (1) is immediate and $d_{\mathcal{F}_{e}}$ is clearly a metric on $\mathcal{F}_{e}$. For the proof of its completeness, let $\left\{u_{n}\right\}_{n \in \mathbb{N}} \subset \mathcal{F}_{e}$ be a Cauchy sequence in $\left(\mathcal{F}_{e}, d_{\mathcal{F}_{e}}\right)$. Noting that $\mathcal{F}$ is dense in $\left(\mathcal{F}_{e}, d_{\mathcal{F}_{e}}\right)$, for each $n \in \mathbb{N}$ take $v_{n} \in \mathcal{F}$ such that $\left\|v_{n}-u_{n}\right\|_{\mathcal{F}_{e}} \leq n^{-1}$. Then $\left\{v_{n}\right\}_{n \in \mathbb{N}}$ is also a Cauchy sequence in $\left(\mathcal{F}_{e}, d_{\mathcal{F}_{e}}\right)$. A Borel-Cantelli argument easily yields a subsequence $\left\{v_{n_{k}}\right\}_{k \in \mathbb{N}}$ of $\left\{v_{n}\right\}_{n \in \mathbb{N}}$ converging $m$-a.e. to some $u \in L^{0}(E, m)$, which means that $u \in \mathcal{F}_{e}$ with approximating sequence $\left\{v_{n_{k}}\right\}_{k \in \mathbb{N}}$ and hence that $\lim _{k \rightarrow \infty}\left\|u-v_{n_{k}}\right\|_{\mathcal{F}_{e}}=0$. The same argument also implies that every subsequence of $\left\{v_{n}\right\}_{n \in \mathbb{N}}$ admits a further subsequence converging to $u$ in $\left(\mathcal{F}_{e}, d_{\mathcal{F}_{e}}\right)$, from which $\lim _{n \rightarrow \infty}\left\|u-v_{n}\right\|_{\mathcal{F}_{e}}=0$ follows. Thus $\lim _{n \rightarrow \infty} \| u-$ $-u_{n} \|_{\mathcal{F}_{e}}=0$.

Lemma 2. (1) $\mathcal{F}_{e} \subset \bigcap_{t \in(0, \infty)} \mathcal{D}\left[T_{t}\right]$ and $T_{t}\left(\mathcal{F}_{e}\right) \subset \mathcal{F}_{e}$ for any $t \in(0, \infty)$.

(2) Let $\eta$ and $\|\cdot\|_{\mathcal{F}_{e}}$ be as in Lemma 1 , and let $u \in \mathcal{F}_{e}$. Then $\mathcal{E}\left(T_{t} u, T_{t} u\right) \leq \mathcal{E}(u, u)$, $\left\|u-T_{t} u\right\|_{2}^{2} \leq t \mathcal{E}(u, u)$ and $\left\|T_{t} u\right\|_{\mathcal{F}_{e}} \leq\left(3+\|\eta\|_{2} \sqrt{t}\right)\|u\|_{\mathcal{F}_{e}}$ for any $t \in(0, \infty), T_{s} T_{t} u=T_{s+t} u$ for any $s, t \in(0, \infty)$, and $\lim _{t \downarrow 0}\left\|u-T_{t} u\right\|_{\mathcal{F}_{e}}=0$.

Proof. Let $\eta,\|\cdot\|_{\mathcal{F}_{e}}$ and $d_{\mathcal{F}_{e}}$ be as in Lemma 1. First we prove (2) for $u \in \mathcal{F}$. The fourth assertion is clear. $T_{t} u \in \mathcal{F}$ and $\mathcal{E}\left(T_{t} u, T_{t} u\right) \leq \mathcal{E}(u, u)$ for $t \in(0, \infty)$ by [6, Lemma 1.3.3-(i)], and $\lim _{t \downarrow 0}\left\|u-T_{t} u\right\|_{\mathcal{F}_{e}}=0$ by [6, Lemma 1.3.3-(iii)]. Let $t \in(0, \infty)$. Noting that $\left\langle f-T_{t} f, T_{t} f\right\rangle=\left\|T_{t / 2} f\right\|_{2}^{2}-\left\|T_{t} f\right\|_{2}^{2} \geq 0$ for $f \in L^{2}(E, m)$, we have $\left\|u-T_{t} u\right\|_{2}^{2}=\langle u-$ $\left.-T_{t} u, u\right\rangle-\left\langle u-T_{t} u, T_{t} u\right\rangle \leq\left\langle u-T_{t} u, u\right\rangle \leq t \mathcal{E}(u, u)$ by [6, Lemma 1.3.4-(i)]. Applying these estimates to $\left\|u-T_{t} u\right\|_{\mathcal{F}_{e}} \leq \mathcal{E}(u, u)^{1 / 2}+\mathcal{E}\left(T_{t} u, T_{t} u\right)^{1 / 2}+\|\eta\|_{2}\left\|u-T_{t} u\right\|_{2}$ easily yields $\left\|T_{t} u\right\|_{\mathcal{F}_{e}} \leq\left(3+\|\eta\|_{2} \sqrt{t}\right)\|u\|_{\mathcal{F}_{e}}$.

Now since $\mathcal{F}$ is dense in a complete metric space $\left(\mathcal{F}_{e}, d_{\mathcal{F}_{e}}\right)$, it follows from the previous paragraph that $\left.T_{t}\right|_{\mathcal{F}}$ is uniquely extended to a continuous map $T_{t}^{e}$ from $\left(\mathcal{F}_{e}, d_{\mathcal{F}_{e}}\right)$ to itself, and then clearly $T_{t}^{e}$ is linear and the assertions of (2) are true with $T_{t}^{e}$ in place of $T_{t}$.

Let $t \in(0, \infty)$ and $u \in \mathcal{F}_{e} \cap L_{+}(E, m)$. It remains to show $T_{t}^{e} u=T_{t} u$, as $v^{+}, v^{-} \in \mathcal{F}_{e}$ for $v \in \mathcal{F}_{e}$. Since $v^{+} \wedge u \in \mathcal{F}_{e} \cap L^{2}(E, m)=\mathcal{F}$ and $\mathcal{E}\left(v^{+} \wedge u, v^{+} \wedge u\right)^{1 / 2} \leq \mathcal{E}(v, v)^{1 / 2}+$ $+\mathcal{E}(u, u)^{1 / 2}$ for any $v \in \mathcal{F}$ by the positivity preserving property of $(\mathcal{E}, \mathcal{F})$, an application of the Banach-Saks theorem [2, Theorem A.4.1-(ii)] assures the existence of an approximating sequence $\left\{w_{n}\right\}_{n \in \mathbb{N}}$ for $u$ such that $0 \leq w_{n} \leq u m$-a.e. A Borel - Cantelli argument yields 
a subsequence $\left\{w_{n_{k}}\right\}_{k \in \mathbb{N}}$ such that $\lim _{k \rightarrow \infty} T_{t} w_{n_{k}}=T_{t}^{e} u m$-a.e., and $T_{t}^{e} u=T_{t} u$ follows by letting $k \rightarrow \infty$ in $T_{t}\left(\inf _{j \geq k} w_{n_{j}}\right) \leq T_{t} w_{n_{k}} \leq T_{t} u m$-a.e.

The following proposition (Proposition 3), which seems new in spite of its easiness, plays an essential role in the proof of $\mathbf{1}) \Rightarrow \mathbf{2}$ ) of Theorem 1. Proposition 3-(2) is an extension of a result of Chen and Kuwae [3, Lemma 3.1] for functions in $\mathcal{F}$ to those in $\mathcal{F}_{e}$, and Proposition 3-(3) extends a basic fact for functions in $\mathcal{F}$ to those in $\mathcal{F}_{e}$.

Proposition 3. (1) Let $u \in \mathcal{F}_{e}$ and $v \in \mathcal{F}$. Then

$$
\lim _{t \downarrow 0} \frac{1}{t}\left\langle u-T_{t} u, v\right\rangle=\mathcal{E}(u, v) \quad \text { and } \quad\left\langle u-T_{t} u, v\right\rangle=\int_{0}^{t} \mathcal{E}\left(u, T_{s} v\right) d s, \quad t \in(0, \infty) .
$$

(2) Let $u \in \mathcal{F}_{e}$. Then $u$ is $\mathcal{E}$-excessive in the wide sense if and only if $\mathcal{E}(u, v) \geq 0$ for any $v \in \mathcal{F} \cap L_{+}(E, m)$, or equivalently, for any $v \in \mathcal{F}_{e} \cap L_{+}(E, m)$.

(3) Let $u \in \mathcal{F}_{e}$. Then $T_{t} u=u$ for any $t \in(0, \infty)$ if and only if $\mathcal{E}(u, u)=0$.

Proof. (1) Let $u \in \mathcal{F}_{e}, v \in \mathcal{F}$ and set $\varphi(t):=\left\langle u-T_{t} u, v\right\rangle$ for $t \in[0, \infty)$, where $T_{0} u:=u$. Then $t^{-1}|\varphi(t)| \leq \mathcal{E}(u, u)^{1 / 2} \mathcal{E}(v, v)^{1 / 2}$ for $t \in(0, \infty)$ and $\lim _{t \downarrow 0} t^{-1} \varphi(t)=\mathcal{E}(u, v)$ if $u \in \mathcal{F}$ by [6, Lemma 1.3.4-(i)], and the same are true for $u \in \mathcal{F}_{e}$ as well by Lemma 2. Using Lemma 2, we easily see also that $\varphi^{\prime}(t)=\mathcal{E}\left(u, T_{t} v\right)$ for $t \in[0, \infty)$ and that $\varphi^{\prime}$ is continuous on $[0, \infty)$, proving $(2.2)$.

(2) The third assertion of Proposition 2 together with the positivity preserving property of $(\mathcal{E}, \mathcal{F})$ easily implies that $\mathcal{E}(u, v) \geq 0$ for any $v \in \mathcal{F} \cap L_{+}(E, m)$ if and only if the same is true for any $v \in \mathcal{F}_{e} \cap L_{+}(E, m)$. The rest of the assertion is immediate from (2.2).

(3) This is an immediate consequence of (2).

The next proposition (Proposition 4), which characterizes the notion of $\mathcal{E}$-excessive functions in terms of $\mathcal{F}_{e}$ and $\mathcal{E}$, is of independent interest. The proof is based on a result [11, Corollary 2.4] of Ouhabaz which provides a characterization of invariance of closed convex sets for semigroups on Hilbert spaces. A similar argument in a more general framework can be found in Shigekawa [14].

Proposition 4. Let $u \in L_{+}(E, m)$. Then $u$ is $\mathcal{E}$-excessive if and only if $v \wedge u \in \mathcal{F}_{e}$ and $\mathcal{E}(v \wedge u, v \wedge u) \leq \mathcal{E}(v, v)$ for any $v \in \mathcal{F}_{e}$.

1. The notion of $\mathcal{E}$-excessive functions is determined solely by the pair $\left(\mathcal{F}_{e}, \mathcal{E}\right)$ of the extended space $\mathcal{F}_{e}$ and the form $\mathcal{E}: \mathcal{F}_{e} \times \mathcal{F}_{e} \rightarrow \mathbb{R}$.

2. Let $u \in L_{+}(E, m)$ be $\mathcal{E}$-excessive and $v \in \mathcal{F}_{e}$. Suppose $u \leq v$ m-a.e. Then $u \in \mathcal{F}_{e}$ and $\mathcal{E}(u, u) \leq \mathcal{E}(v, v)$.

Remark 3. Chen and Kuwae [3, Lemma 3.3] gave a probabilistic proof of Corollary 2 for the Dirichlet forms associated with symmetric right Markov processes.

Proof of Proposition 4. Let $K_{u}:=\left\{f \in L^{2}(E, m) \mid f \leq u m\right.$-a.e. $\}$, which is clearly a closed convex subset of $L^{2}(E, m)$. We claim that

$$
u \text { is } \mathcal{E} \text {-excessive if and only if } T_{t}\left(K_{u}\right) \subset K_{u} \text { for any } t \in(0, \infty) .
$$

Indeed, let $t \in(0, \infty)$. If $T_{t} u \leq u m$-a.e. then $T_{t} f \leq T_{t} u \leq u m$-a.e. for any $f \in K_{u}$ and hence $T_{t}\left(K_{u}\right) \subset K_{u}$. Conversely if $T_{t}\left(K_{u}\right) \subset K_{u}$, then choosing $\eta \in L^{2}(E, m)$ so that $\eta>0$ $m$-a.e., we have $(n \eta) \wedge u \uparrow u m$-a.e., $(n \eta) \wedge u \in K_{u}$ and hence $T_{t} u=\lim _{n \rightarrow \infty} T_{t}((n \eta) \wedge u) \leq u$ $m$-a.e. 
On the other hand, since the projection of $f \in L^{2}(E, m)$ on $K_{u}$ is given by $f \wedge u$, [11, Corollary 2.4] tells us that $T_{t}\left(K_{u}\right) \subset K_{u}$ for any $t \in(0, \infty)$ if and only if

$$
v \wedge u \in \mathcal{F} \quad \text { and } \quad \mathcal{E}(v \wedge u, v \wedge u) \leq \mathcal{E}(v, v) \quad \text { for any } v \in \mathcal{F}
$$

Finally, $\mathcal{F}_{e} \cap L^{2}(E, m)=\mathcal{F}$ and Proposition 2 easily imply that (2.4) is equivalent to the same condition with $\mathcal{F}_{e}$ in place of $\mathcal{F}$, completing the proof.

\section{Proof of Theorem 1}

We are now ready for the proof of Theorem 1. We assume throughout this section that our closed symmetric form $(\mathcal{E}, \mathcal{F})$ is a Dirichlet form. The proof consists of three steps. The first one is Proposition 5 below, which establishes $\mathbf{1}) \Rightarrow \mathbf{2}$ ) of Theorem 1 and whose proof makes full use of Proposition 3-(3). Recall the following notions concerning the irreducibility of $(\mathcal{E}, \mathcal{F})$; see [6, Section 1.6] or [2, Section 2.1] for details.

Definition 5. (1) $A$ set $A \in \mathcal{B}$ is called $\mathcal{E}$-invariant if and only if $\mathbf{1}_{A} T_{t}\left(f \mathbf{1}_{E \backslash A}\right)=0 \mathrm{~m}$-a.e. for any $f \in L^{2}(E, m)$ and any $t \in(0, \infty)$.

(2) $(\mathcal{E}, \mathcal{F})$ is called irreducible if and only if either $m(A)=0$ or $m(E \backslash A)=0$ holds for any $\mathcal{E}$-invariant $A \in \mathcal{B}$.

Lemma 3. Let $u \in L_{+}(E, m)$ be $\mathcal{E}$-excessive. Then $\{u=0\}$ is $\mathcal{E}$-invariant.

Proof. In fact, the following proof is valid as long as $(\mathcal{E}, \mathcal{F})$ is a symmetric positivity preserving form. Let $B:=\{u=0\}, f \in L^{2}(E, m)$ and set $f_{n}:=|f| \wedge(n u)$ for $n \in \mathbb{N}$, so that $f_{n} \uparrow|f| \mathbf{1}_{E \backslash B} m$-a.e. Then $0 \leq \mathbf{1}_{B} T_{t} f_{n} \leq \mathbf{1}_{B} T_{t}(n u) \leq n \mathbf{1}_{B} u=0 \mathrm{~m}$-a.e., and letting $n \rightarrow \infty$ leads to $\left|\mathbf{1}_{B} T_{t}\left(f \mathbf{1}_{E \backslash B}\right)\right| \leq \mathbf{1}_{B} T_{t}\left(|f| \mathbf{1}_{E \backslash B}\right)=0 \mathrm{~m}$-a.e. Thus $B=\{u=0\}$ is $\mathcal{E}$-invariant.

Proposition 5. Suppose that $(\mathcal{E}, \mathcal{F})$ is irreducible. If $u \in \mathcal{F}_{e}$ and $\mathcal{E}(u, u)=0$ then $u \in \mathbb{R} \mathbf{1}$.

Proof. We follow [2, Proof of Theorem 2.1.11, (i) $\Rightarrow$ (ii)]. Let $u \in \mathcal{F}_{e}$ satisfy $\mathcal{E}(u, u)=0$. We may assume that $m(\{u>0\})>0$. Let $\lambda \in[0, \infty)$ and $u_{\lambda}:=u-u \wedge \lambda$. Since $(\mathcal{E}, \mathcal{F})$ is assumed to be a Dirichlet form, $u_{\lambda} \in \mathcal{F}_{e} \cap L_{+}(E, m)$ and $\mathcal{E}\left(u_{\lambda}, u_{\lambda}\right)=0$ (see Proposition 4 ), and therefore $T_{t} u_{\lambda}=u_{\lambda}$ for any $t \in(0, \infty)$ by Proposition $3-(3)$. Then $\left\{u_{\lambda}=0\right\}$ is $\mathcal{E}$-invariant by Lemma 3 , and the irreducibility of $(\mathcal{E}, \mathcal{F})$ implies that either $m\left(\left\{u_{\lambda}=0\right\}\right)=0$ or $m\left(\left\{u_{\lambda}>0\right\}\right)=0$ holds. Now setting $\kappa:=\sup \left\{\lambda \in[0, \infty) \mid m\left(\left\{u_{\lambda}=0\right\}\right)=0\right\}$, we easily see that $\kappa \in(0, \infty)$ and that $u=\kappa m$-a.e.

For the rest of the proof of Theorem 1, let us recall basic notions concerning recurrence and transience of Dirichlet forms. See [6, Sections 1.5 and 1.6] or [2, Section 2.1] for details. For $t \in(0, \infty)$, we define $S_{t}: L^{2}(E, m) \rightarrow L^{2}(E, m)$ by $S_{t} f:=\int_{0}^{t} T_{s} f d s$, where the integral is the Riemann integral in $L^{2}(E, m)$. Then $t^{-1} S_{t}$ is a Markovian symmetric bounded linear operator on $L^{2}(E, m)$, and therefore it is canonically extended to an operator on $L_{+}(E, m)$ by Proposition 1. Furthermore, for any $s, t \in(0, \infty)$ we easily see that $S_{s+t}=S_{s}+T_{s} S_{t}=S_{s}+S_{t} T_{s}$ as operators on $L_{+}(E, m)$ or on $L^{2}(E, m)$.

Let $f \in L_{+}(E, m)$. Then $0 \leq S_{s} f \leq S_{t} f m$-a.e. and $0 \leq G_{\beta} f \leq G_{\alpha} f m$-a.e. for $0<s<t, 0<\alpha<\beta$. Therefore there exists a unique $G f \in L_{+}(E, m)$ satisfying $S_{N} f \uparrow G f m$-a.e. It is immediate that $G f_{n} \uparrow G f m$-a.e. for any $\left\{f_{n}\right\}_{n \in \mathbb{N}} \subset L_{+}(E, m)$ with $f_{n} \uparrow f m$-a.e. Since, on $L^{2}(E, m),\left\{G_{\alpha}\right\}_{\alpha \in(0, \infty)}$ is the Laplace transform of $\left\{T_{t}\right\}_{t \in(0, \infty)}$, we see that $S_{t_{n}} f \uparrow G f m$-a.e. and $G_{\alpha_{n}} f \uparrow G f m$-a.e. for any $\left\{t_{n}\right\}_{n \in \mathbb{N}},\left\{\alpha_{n}\right\}_{n \in \mathbb{N}} \subset(0, \infty)$ with 
$t_{n} \uparrow \infty, \alpha_{n} \downarrow 0$. Moreover, since $S_{t+N} f=S_{t} f+T_{t} S_{N} f \geq T_{t} S_{N} f m$-a.e. for $t \in(0, \infty)$ and $N \in \mathbb{N}$, by letting $N \rightarrow \infty$ we have $T_{t} G f \leq G f m$-a.e., that is, $G f$ is $\mathcal{E}$-excessive. We call this operator $G: L_{+}(E, m) \rightarrow L_{+}(E, m)$ the 0 -resolvent associated with $(\mathcal{E}, \mathcal{F})$.

Definition 6 (Transience and Recurrence). (1) $(\mathcal{E}, \mathcal{F})$ is called transient if and only if $G f<\infty m$-a.e. for some $f \in L_{+}(E, m)$ with $f>0 m$-a.e.

(2) $(\mathcal{E}, \mathcal{F})$ is called recurrent if and only if $m(\{0<G f<\infty\})=0$ for any $f \in L_{+}(E, m)$.

By [6, Lemma 1.5.1], $(\mathcal{E}, \mathcal{F})$ is transient if and only if $G f<\infty m$-a.e. for any $f \in L_{+}^{1}(E, m)$. On the other hand, by [6, Theorem 1.6.3], $(\mathcal{E}, \mathcal{F})$ is recurrent if and only if $\mathbf{1} \in \mathcal{F}_{e}$ and $\mathcal{E}(\mathbf{1}, \mathbf{1})=0$.

The following proposition is the second step of the proof of Theorem 1 .

Proposition 6. Assume that $(\mathcal{E}, \mathcal{F})$ is recurrent. If $u \in L_{+}^{0}(E, m)$ is $\mathcal{E}$-excessive then $u \in \mathcal{F}_{e}$ and $\mathcal{E}(u, u)=0$.

Proof. Let $n \in \mathbb{N}$. Then $u \wedge n \leq n \mathbf{1} m$-a.e., $n \mathbf{1} \in \mathcal{F}_{e}$ and $\mathcal{E}(n \mathbf{1}, n \mathbf{1})=0$ by the recurrence of $(\mathcal{E}, \mathcal{F})$, and $u \wedge n$ is $\mathcal{E}$-excessive since so are $u$ and $\mathbf{1}$. Thus $u \wedge n \in \mathcal{F}_{e}$ and $\mathcal{E}(u \wedge n, u \wedge n)=0$ by Corollary 2. Lemma 1-(2) implies that $\lim _{n \rightarrow \infty}\|v-u \wedge n\|_{\mathcal{F}_{e}}=0$ for some $v \in \mathcal{F}_{e}$ with $\|\cdot\|_{\mathcal{F}_{e}}$ as defined there, and then we easily have $u=v \in \mathcal{F}_{e}$ and $\mathcal{E}(u, u)=0$.

As the third step, now we finish the proof of Theorem 1 .

Proof of Theorem 1. 1) $\Rightarrow$ 2) follows by Proposition 5, and so does $\mathbf{1}) \Rightarrow$ 5) by Propositions 5 and 6 . 2) $\Rightarrow \mathbf{3}$ ), 4) $\Rightarrow \mathbf{6}$ ) and 5) $\Rightarrow \mathbf{6}$ ) are trivial.

1) $\Rightarrow$ 4): Let $u \in \mathcal{F}_{e}$ be $\mathcal{E}$-excessive in the wide sense, $n \in \mathbb{N}$ and $u_{n}:=u \wedge n$. Then $u_{n} \in \mathcal{F}_{e}, u_{n}$ is also $\mathcal{E}$-excessive in the wide sense, $n \mathbf{1}-u_{n} \in \mathcal{F}_{e} \cap L_{+}(E, m)$ and hence $\mathcal{E}\left(u_{n}, u_{n}\right)=\mathcal{E}\left(u_{n}, u_{n}-n \mathbf{1}\right) \leq 0$ by Proposition 3-(2). As in the proof of Proposition 6, letting $n \rightarrow \infty$ we get $\mathcal{E}(u, u)=0$ by Lemma $1-(2)$, and hence $u \in \mathbb{R} \mathbf{1}$ by Proposition 5 .

3) $\Rightarrow \mathbf{1}):(\mathcal{E}, \mathcal{F})$ is recurrent since $\mathbf{1} \in \mathcal{F}_{e}$ and $\mathcal{E}(\mathbf{1}, \mathbf{1})=0$. Let $A \in \mathcal{B}$ be $\mathcal{E}$-invariant. Then $\mathbf{1}_{A}=\mathbf{1}_{A} \mathbf{1} \in \mathcal{F}_{e} \cap L_{+}^{\infty}(E, m)$ and $0 \leq \mathcal{E}\left(\mathbf{1}_{A}, \mathbf{1}_{A}\right) \leq \mathcal{E}(\mathbf{1}, \mathbf{1})=0$ by [6, Theorem 1.6.1]. Now 3) implies $\mathbf{1}_{A} \in \mathbb{R} \mathbf{1}$, and hence either $m(A)=0$ or $m(E \backslash A)=0$.

6) $\Rightarrow 3)$ when $(E, \mathcal{B}, m)$ is non-trivial: Choose $g \in L^{1}(E, m)$ so that $g>0 m$-a.e., and set $E_{c}:=\{G g=\infty\}$. Then $\mathbf{1}_{E_{c}} \in \mathcal{F}_{e} \cap L_{+}^{\infty}(E, m)$ and $\mathcal{E}\left(\mathbf{1}_{E_{c}}, \mathbf{1}_{E_{c}}\right)=0$ by [6, Corollary 1.6.2], and 6) together with Proposition 3-(3) implies $\mathbf{1}_{E_{c}} \in \mathbb{R} \mathbf{1}$, i.e., either $m\left(E_{c}\right)=0$ or $m\left(E \backslash E_{c}\right)=0$. In view of 6) and Proposition 3-(3), it suffices to show $m\left(E \backslash E_{c}\right)=0$.

Suppose $m\left(E_{c}\right)=0$, so that $(\mathcal{E}, \mathcal{F})$ is transient, and set $\eta:=g /(1 \vee G g)$. Then $0<\eta \leq$ $\leq g m$-a.e. and $\langle\eta, G \eta\rangle \leq\langle g /(1 \vee G g), G g\rangle \leq\|g\|_{1}<\infty$. Let $f \in L_{+}^{1}(E, m) \cap L^{2}(E, m)$ and set $f_{n}:=f \wedge(n \eta)$ for $n \in \mathbb{N}$. Then $f_{n} \in L_{+}^{2}(E, m), G f_{n} \leq n G \eta<\infty m$-a.e., $\left\langle f_{n}, G f_{n}\right\rangle<\infty$ and $f_{n} \uparrow f m$-a.e. Since $\mathcal{E}\left(G_{\alpha} f_{n}, G_{\alpha} f_{n}\right) \leq\left\langle f_{n}, G_{\alpha} f_{n}\right\rangle \leq\left\langle f_{n}, G f_{n}\right\rangle<\infty$ for $\alpha \in(0, \infty)$, Proposition 2 implies $G f_{n} \in \mathcal{F}_{e}$. Since $G f_{n}$ is $\mathcal{E}$-excessive, so is $n \wedge G f_{n} \in \mathcal{F}_{e} \cap L_{+}^{\infty}(E, m)$ and 6) yields $n \wedge G f_{n} \in \mathbb{R} \mathbf{1}$. Letting $n \rightarrow \infty$ and noting $G f<\infty m$-a.e. by the transience of $(\mathcal{E}, \mathcal{F})$, we get $G f \in \mathbb{R} \mathbf{1}$. Let $\alpha \in(0, \infty)$. Then $G_{\alpha} f \in L_{+}^{1}(E, m) \cap L^{2}(E, m)$ and hence $G G_{\alpha} f \in \mathbb{R} \mathbf{1}$. Letting $n \rightarrow \infty$ in $G_{\alpha} f=G_{1 / n} f-(\alpha-1 / n) G_{1 / n} G_{\alpha} f$ implies that $G_{\alpha} f=G f-\alpha G G_{\alpha} f \in \mathbb{R} 1$. Since $\alpha G_{\alpha} f \rightarrow f$ in $L^{2}(E, m)$ as $\alpha \rightarrow \infty$, we conclude that $L_{+}^{1}(E, m) \cap L^{2}(E, m) \subset \mathbb{R} \mathbf{1}$, contradicting the assumption that $(E, \mathcal{B}, m)$ is non-trivial. Thus $m\left(E \backslash E_{c}\right)=0$ follows. 


\section{Acknowledgements}

The author would like to express his deepest gratitude toward Professor Masatoshi Fukushima for fruitful discussions and for having suggested this problem to him in [5]. The author would like to thank Professor Masanori Hino for detailed valuable comments on the proofs in an earlier version of the manuscript; in particular, the proofs of Propositions 3 and 6 have been much simplified by following his suggestion of the use of Lemma 1 and Corollary 2. The author would like to thank also Professor Masayoshi Takeda and Professor Jun Kigami for valuable comments.

\section{REMARK}

${ }^{1}$ JSPS Research Fellow PD (20.6088): The author was supported by the Japan Society for the Promotion of Science.

\section{REFERENCES}

1. Blumenthal R.M., Getoor R.K. Markov Processes and Potential Theory. New York, Academic Press, 1968. 312 p.

2. Chen Z.-Q., Fukushima M. Symmetric Markov Processes, Time Change, and Boundary Theory. Princeton, Princeton University Press, 2012. 512 p.

3. Chen Z.-Q., Kuwae K. On subharmonicity for symmetric Markov processes. J. Math. Soc. Japan, 2012, vol. 64, pp. 1181-1209.

4. Fukushima M. On extended Dirichlet spaces and the space of BL functions. Potential theory and stochastics in Albac, Theta Ser. Adv. Math.. Bucharest, Theta, 2009, vol. 11, pp. 101-110.

5. Fukushima M. Personal communication. (December 17, 2008).

6. Fukushima M., Oshima Y., Takeda M. Dirichlet Forms and Symmetric Markov Processes. Berlin, Walter de Gruyter, 2011. 489 p.

7. Fukushima M., Takeda M. Markov Processes. Tokyo, Baifukan, 2008. 489 p. (in Japanese)

8. Getoor R.K. Excessive Measures. Boston, Birkhäuser, 1990. 190 p.

9. Getoor R.K. Transience and recurrence of Markov processes. Séminaire de probabilités de Strasbourg. Berlin, Springer, 1980, vol. 14, pp. 397-409.

10. Oshima Y. Potential of recurrent symmetric Markov processes and its associated Dirichlet spaces. Functional analysis in Markov processes (Katata/Kyoto, 1981), Lecture Notes in Math.. Berlin, Springer, 1982, vol. 923, pp. 260-275.

11. Ouhabaz E.M. Invariance of Closed Convex Sets and Domination Criteria for Semigroups. Potential Anal., 1996, vol. 5, pp. 611-625.

12. Schmuland M. Extended Dirichlet spaces. C. R. Math. Acad. Sci. Soc. R. Can., 1999, vol. 21, pp. 146-152.

13. Schmuland M. Positivity preserving forms have the Fatou property. Potential Anal., 1999, vol. 10, pp. 373-378.

14. Shigekawa I. Semigroups preserving a convex set in a Banach space. Kyoto J. Math., 2011, vol. 51, pp. 647-672. 


\section{ЭКВИВАЛЕНТНОСТЬ РЕКУРРЕНТНОСТИ И ЛИУВИЛЛЕВА СВОЙСТВА ДЛЯ СИММЕТРИЧНЫХ ФОРМ ДИРИХЛЕ}

\section{Наотака Кадзино}

Доцент, Факультет математики, Высшая естественнонаучная школа, Kobe University nkajino@math.kobe-u.ac.jp

Rokkodai-cho 1-1, Nada-ku, Kobe 657-8501, Japan

Аннотация. Рассмотрим симметричную форму Дирихле $(\mathcal{E}, \mathcal{F})$ на $\sigma$ конечном (нетривиальном) метрическом пространстве $(E, \mathcal{B}, m)$ с ассоциированной марковской полугруппой $\left\{T_{t}\right\}_{t \in(0, \infty)}$. В работе доказано, что $(\mathcal{E}, \mathcal{F})$ несократимая и рекуррентная тогда и только тогда, когда не существует непостоянной $\mathcal{B}$-измеримой и $\mathcal{E}$-эксцессивной функции $u: E \rightarrow[0, \infty]$, то есть такой, что $T_{t} u \leq u m$-а.е. для всех $t \in(0, \infty)$. Так же доказано, что эти условия эквивалентны равенству $\left\{u \in \mathcal{F}_{e} \mid \mathcal{E}(u, u)=0\right\}=\mathbb{R} \mathbf{1}$, где $\mathcal{F}_{e}$ означает расширенное пространство Дирихле, ассоциированное с $(\mathcal{E}, \mathcal{F})$. Доказательство чисто аналитическое и не требует дополнительных ограничений на фазовое пространство и форму. В процессе доказательства так же представлена характеристика $\mathcal{E}$-эксцессивности в терминах $\mathcal{F}_{e}$ и $\mathcal{E}$, которая справедлива для любой симметричной формы, сохраняющей положительность.

Ключевые слова: симметричные формы Дирихле; симметричные формы, сохраняющие положительность; расширенное пространство Дирихле, эксцессивные функции, рекуррентность, лиувиллево свойство. 